\title{
AÇÕES INTERDISCIPLINARES EM SAÚDE, ESTRATÉGIAS DE ENSINO EM TEMPOS DE PANDEMIA: RELATO DE EXPERIÊNCIA ${ }^{1}$
}

\author{
INTERDISCIPLINARY ACTIONS IN HEALTH, TEACHING STRATEGIES IN \\ PANDEMIC TIMES: EXPERIENCE REPORT
}

\author{
Lenise Menezes Seerig ${ }^{2}$, Liliane Alves Pereira ${ }^{3}$, Morgana Christmann ${ }^{4}$, \\ Letícia Fernandez Frigo ${ }^{5}$, Camila Lehnhart $\operatorname{Vargas}^{6}$ e Aline Krüger Batista ${ }^{7}$
}

\section{RESUMO}

Este trabalho tem como objetivo relatar as ações do subprojeto de Ensino-Extensão Ações Extensionistas Interdisciplinares em Saúde, dos cursos de Enfermagem, Fisioterapia, Nutrição e Odontologia da Universidade Franciscana-Santa Maria/RS, composto pelas disciplinas Ações Interdisciplinares em Saúde, Humanização na Prática Odontológica e Ações Integradas em Odontologia I, durante o $1^{\mathrm{a}}$ semestre/2020, na pandemia da COVID-19 em que as aulas tiveram que ser essencialmente no ambiente virtual. Como ferramenta metodológica, para o desenvolvimento das atividades das disciplinas componentes, foi utilizada a Espiral Construtivista. Foram realizadas vídeo aulas síncronas e eventualmente assíncronas, disponibilizados materiais de leitura, discussão virtual pelo ambiente virtual Moodle/Microsoft Teams, vídeos com profissionais atuantes na rede de atenção à saúde e envio de materiais educativos aos projetos sociais e às ESF e UBS da cidade. A situação pandêmica trouxe tanto para os estudantes, quanto para os docentes o desafio de incorporar na sua prática atividades remotas mediadas pelas tecnologias, substituindo a visão tradicional de ensinar a aprender, que há tempos discute-se ser necessária superar.

Palavras-chave: COVID-19; Equipe Interdisciplinar de Saúde; Extensão Comunitária.

\section{ABSTRACT}

This article aims to report the actions of the teaching-extension Interdisciplinary Extension Actions in Health subproject of the Nursing, Physiotherapy, Nutrition and Dentistry courses during 1st semester/2020 in the COVID 19 pandemic which classes had to be essentially in the virtual environment. As a methodological strategy for development of activities in the component disciplines the constructivist Spiral was used. Synchronous and eventually asynchronous video lessons were held Reading materials and made available virtual discussions through

1 Relato de Experiência

2 Graduada em Odontologia pela Universidade Federal de Santa Maria, Mestre e Doutora em Epidemiologia Universidade Federal de Pelotas. Docente da Universidade Franciscana. E-mail: lenise.seerig@ufn.edu.br

3 Graduada em Enfermagem, pelo Instituto Doctum de Educação e Tecnologia, Mestre e Doutora em Enfermagem pela Universidade Federal de Rio Grande. Docente da Universidade Franciscana. E-mail: liliane.pereira@ufn.edu.br

4 Graduada em Fisioterapia pela Universidade Feevale, Mestre e Doutora em Educação pela Universidade Federal de Santa Maria. Docente da Universidade Franciscana. E-mail: morgana.christmann@ufn.edu.br

5 Graduada em Fisioterapia pela Universidade Franciscana, Mestre e Doutora em Distúrbios da Comunicação Humana pela Universidade Federal de Santa Maria. Docente da Universidade Franciscana. E-mail: leticiafrigo@ufn.edu.br 6 Graduada em Nutrição pela Universidade Franciscana, Mestre e Doutora em Distúrbios da Comunicação Humana pela Universidade Federal de Santa Maria. Docente da Universidade Franciscana. E-mail: camila.lehnhart@ufn.edu.br 7 Graduada em Odontologia pela Universidade Federal de Santa Maria, Mestre em Ciências Odontológicas com ênfase em Saúde Coletiva pela Universidade Federal de Santa Maria. Docente da Universidade Franciscana. E-mail: aline.kruger@ufn.edu.br 
environment Moodle/Microsoft teams, videos with professionals working and health care network and educational materials are sent to social projects and to the ESF and UBS in the city. The pandemic situation brought both students and teachers the challenge of incorporating remote activities mediated by technologies into their practice, replacing the traditional view of teaching how to learn, which has long been discussed to be necessary to overcome.

Keywords: COVID-19, Interdisciplinary Health Team, Community-Institutional Relations.

\section{INTRODUÇÃO}

As Diretrizes Curriculares Nacionais (DCN) reforçam nitidamente a importância do trabalho em equipes multiprofissionais, entendendo que a "equipe de saúde como um todo tem maior impacto do que a soma das contribuições de forma isolada". A inadequação da formação dos profissionais de saúde tem levado a necessidade de requalificação profissional que podem ser constatadas através de recursos gastos pelo Ministério da Saúde em “capacitações”. A implantação das DCN nas atividades teórico/práticas procura consolidar as diretrizes e princípios, assumindo, portanto, grande importância no aperfeiçoamento do Sistema Único de Saúde (SUS) (MORITA, 2003).

No campo da saúde, a extrema fragmentação do conhecimento tem demonstrado sua insuficiência e colocado a multidisciplinaridade no centro das discussões acerca do desenvolvimento das ciências e das práticas sanitárias. A influência do modelo fragmentado de organização de trabalho, em que cada profissional realiza parcelas do trabalho sem uma integração com as demais áreas envolvidas, tem sido apontada como uma das razões que dificultam a realização de um trabalho em saúde mais integrador e de melhor qualidade, tanto na perspectiva daqueles que o realizam como para aqueles que dele usufruem (MATOS; PIRES; SOUSA, 2010).

Considerando a realidade e as especificidades do trabalho em saúde, que é desenvolvido por seres humanos para outros seres humanos, cuja complexidade ultrapassa os saberes de uma única profissão, é que tem se defendido que o trabalho em saúde deve envolver práticas multiprofissionais por uma necessidade própria da evolução do conhecimento, do desenvolvimento profissional e da complexidade crescente dos problemas de saúde na atualidade (MATOS; PIRES; SOUSA, 2010).

Este artigo, portanto, tem como objetivo relatar as ações do subprojeto de Ensino-Extensão Ações Extensionistas Interdisciplinares em Saúde, dos cursos de Enfermagem, Fisioterapia, Nutrição e Odontologia da Universidade Franciscana-Santa Maria/RS, composto pelas disciplinas Ações interdisciplinares em saúde, Humanização na Prática Odontológica e Ações Integradas em Odontologia I, durante o $1^{\text {a }}$ semestre/2020, na pandemia da COVID-19 em que as aulas tiveram que ser essencialmente no ambiente virtual.

O objetivo do referido subprojeto é promover ações de promoção de saúde junto à comunidade do território de abrangência da Estratégia da Saúde da Família (ESF), possibilitar a reflexão do aluno acerca da interprofissionalidade, do princípio da equidade e dos determinantes sociais, familiarizar o 
acadêmico com o processo de trabalho dos diferentes profissionais da saúde a partir do conhecimento das bases psicológicas do comportamento humano e suas implicações para a saúde, bem como conhecer os instrumentos de promoção de saúde das coletividades, especialmente as visitas domiciliares e interação do tripé ensino, pesquisa e extensão como construção do ser profissional. Ainda, torna-se relevante que essa indução para a reflexão multiprofissional se dê já desde o primeiro semestre das graduações.

\section{METODOLOGIA}

Este artigo trata de um relato da experiência docente. Segundo Prodanov e Freitas (2013, p. 158), um relatório resultante das ações desenvolvidas em projetos, "visa pura e simplesmente a historiar seu desenvolvimento, muito mais no sentido de apresentar os caminhos percorridos, de descrever as atividades realizadas e de apreciar os resultados".

Para o subprojeto foi utilizada, como ferramenta metodológica para o desenvolvimento das atividades das disciplinas componentes, a Espiral Construtivista, proposta por Lima (2017). Tal metodologia ativa tem como proposta identificar os problemas, formular explicações, elaborar questões, buscar novas informações, construir novos significados e avaliar os processos. Com isso, os estudantes junto aos professores, profissionais da saúde dos serviços e agente comunitário de saúde (ACS), socializam as dificuldades encontradas frente às pessoas da comunidade, propondo soluções de acordo com a realidade local.

A Espiral Construtivista é uma metodologia ativa de ensino aprendizagem, que se baseia em disparadores, utilizando-se da contextualização da situação, com enfoque crítico e reflexivo na busca de soluções para a situação (LIMA, 2017). Vale mencionar que para este subprojeto, optou-se por diversificar as metodologias, buscando abranger as expectativas dos estudantes e contemplar o conteúdo previsto nas disciplinas que precisaram ser adaptadas à realidade atual da pandemia da COVID-19, com o ensino de forma remota. Entretanto, esse redimensionamento das atividades continuou primando pelo objetivo principal das disciplinas através das metodologias ativas. Nesta adaptação, a centralidade do acadêmico como protagonista das reflexões tornou-se ainda mais evidente, sendo estimulado a buscar junto aos territórios das diversas comunidades em que estão inseridos os enfrentamentos vivenciados pelos profissionais de saúde das diversas áreas durante a atuação no cenário da pandemia.

\section{RESULTADOS}

A disciplina de Ações Interdisciplinares em Saúde é ofertada no primeiro semestre dos cursos de Enfermagem, Fisioterapia, Nutrição e Odontologia. Participaram 140 estudantes que foram distribuídos em equipes de trabalho, as quais foram nominadas pelos mesmos, buscando caracterizar 
a equipe. Estas eram compostas por estudantes dos diferentes cursos, proporcionando a integração entre as equipes interdisciplinares. As atividades iniciais foram presenciais, sendo que a primeira atividade teve como objetivo o trabalho em equipe com a dinâmica do desenho de um animal, neste caso uma Vaca.

Esta dinâmica é interessante e divertida, busca integrar os estudantes. Propõe estimular os estudantes a construir um desenho de maneira compartilhada sem haver interação direta em cada peça desenhada, uma vez que cada parte do corpo do animal deve ser desenhado por uma equipe, para somente no final unir as partes que formam a ilustração. O objetivo desta atividade além de integrar, é que os estudantes percebam que cada pessoa, ou grupo, tem uma forma diferente de enxergar a proposta e que com o trabalho em equipe, cada um contribuindo com uma parte da atividade é possível construir o todo.

Entretanto, a partir do momento em que se iniciou o distanciamento social, foram propostas diversas atividades remotas, buscando diversificar as metodologias com base em adaptações das atividades já idealizadas. Dentre estas foram realizadas vídeo aulas síncronas e eventualmente assíncronas, disponibilizados materiais de leitura, discussão virtual pelo ambiente virtual Moodle/Microsoft Teams, vídeos com profissionais atuantes na rede de atenção à saúde da cidade de Santa Maria. Buscando formas de diversificar as atividades e tornar a disciplina atrativa no contexto de uma pandemia, os estudantes, participaram em uma Webinar da Faculdade de Saúde Pública da Universidade de São Paulo com subsequente fórum de discussão da atividade, a construção de uma linha do tempo sobre as epidemias mundiais, e puderam vivenciar junto aos profissionais de saúde de seus municípios e equipes atuantes em projetos de auxílio à comunidade em momento de crise, atitudes que estão sendo realizadas em tempos da pandemia do COVID-19. Salienta-se que os estudantes, foram instigados a utilizar da criatividade para a construção dos seus trabalhos e por isso os diferentes recursos digitais foram explorados pelos mesmos.

Os trabalhos extensionistas foram apresentados na plataforma virtual Moodle/Microsoft Teams da Universidade Franciscana (UFN). Quanto à abrangência, consideramos a possibilidade de compartilhamento das ações entre os estudantes e destes com seus municípios, fazendo com que o conhecimento ficasse amplamente difundido. Salienta-se que as atividades foram realizadas em equipe interprofissional o que permitiu um diálogo virtual e assertivo dos estudantes com suas equipes resultando na partilha já mencionada. Além disso, foram desenvolvidas atividades que incentivam a autonomia dentro de uma equipe formada por estudantes de diferentes núcleos da saúde. Essas atividades permitiram que cada aluno e cada profissional de saúde também pudesse fortalecer o caráter identitário da profissão através e a partir do diálogo interprofissional.

A disciplina de Humanização na Prática Odontológica tem caráter interdisciplinar por ser ministrada no segundo semestre do curso de Odontologia por uma professora do curso de psicologia e uma professora do curso de odontologia. O objetivo da disciplina é capacitar os futuros odontólogos 
no reconhecimento da situação social, econômica, cultural, política e educacional da população brasileira, habilitando-o assim para o diagnóstico e apreensão da realidade e do trabalho em saúde, como um todo, a partir do conhecimento das bases sociológicas, éticas e psicológicas do comportamento humano e suas implicações para a saúde. Também prevê desenvolver habilidades e competências, capacitando o estudante para executar educação em saúde bucal, baseando em orientações de higiene oral no paciente infantil. No referido semestre, participaram da disciplina 26 acadêmicos e em torno de 50 crianças do projeto social Golfe na Escola foram beneficiadas com kits de escovação e cartas explicativas sobre higiene bucal produzidas pelos estudantes. De forma remota os estudantes receberam preparo teórico sobre aspectos psicológicos do atendimento odontológico e criação de vínculo e acolhimento, e foi solicitado que cada um realizasse um treinamento em escovação supervisionada em um integrante da sua família. As competências dessa disciplina foram atingidas através do desenvolvimento da habilidade de realizar técnicas de escovação e de produzir uma carta com a intenção de criar educação em saúde na forma de escrita, com linguagem acessível para as crianças.

A disciplina de Ações Integradas em Odontologia I tem por objetivo estimular o raciocínio crítico do estudante frente à realidade de uma população dotada de toda sua complexidade social, econômica e cultural e que necessita de atenção odontológica, fazendo-o identificar seus problemas e propor soluções criativas, além de possibilitar que o estudante vivencie as dificuldades, desafios e ações em educação e promoção de saúde. Espera-se que o estudante desenvolva a habilidade de se comunicar em equipe, a ter autonomia e uma visão reflexiva. No primeiro semestre de 2020, a disciplina contou com 45 alunos do terceiro semestre de Odontologia. Foram realizados infográficos por ciclo de vida, que foram disponibilizados para as ACS distribuírem no território da ESF Urlândia, a qual abrange em torno de 3000 pessoas. Adicionalmente, foram preparados vídeos de promoção de saúde para adolescentes, os quais forma disponibilizados na FASE (Fundação de atendimento socioeducativo do Rio Grande do Sul) para os adolescentes lá internados (em número de 55). Visando buscar a visualização do enfrentamento da pandemia pelos profissionais da Odontologia de diversos municípios do estado do RS (aqueles de residência dos acadêmicos), foi proposta uma atividade de extensão a ser realizada por telefone ou presencialmente (conforme disponibilidade dos dentistas) nas ESF ou UBS, onde puderam trazer as informações de cada local e compartilhar estas experiências em uma web conferência com os colegas.

A situação pandêmica trouxe tanto para os estudantes, quanto para os docentes o desafio de incorporar na sua prática atividades remotas mediadas pelas tecnologias, substituindo a visão tradicional de ensinar a aprender, que há tempos discute-se ser necessária superar.

\section{DISCUSSÃO}

As disciplinas com caráter extensionista têm sido um recurso importante para a aproximação dos estudantes com a realidade que é vivenciada na prática dos profissionais da área da saúde, 
vivenciando a interdisciplinaridade e interprofissionalidade. Tais estratégias são importantes para a formação dos profissionais da saúde, uma vez que segundo Feuerwerker e Cecílio (2007). o avanço tecnológico e a especialização foram fatores que influenciaram significativamente a formação dos profissionais num modelo reducionista e fragmentado nos últimos anos.

A atuação interdisciplinar é preconizada para a abordagem nos diferentes espaços que compõem a rede de atenção à saúde, pelas políticas de saúde brasileiras e incentivada no processo formativo dos estudantes pelas Diretrizes Curriculares Nacionais dos diferentes cursos da área. Estes são elementos que merecem atenção das Instituições de Ensino Superior (IES), proporcionando a qualificação em espaços onde os estudantes possam interagir com colegas de outras profissões, trabalhando de maneira cooperativa, colaborativa e interprofissional e por conseguinte qualificando a atenção prestada aos seus usuários (FARIAS; RIBEIRO; ANJOS et al., 2018).

Além disso, a característica destas disciplinas, que se propõe a aproximar-se da comunidade e produzir mudanças efetivas nos locais que acessa, sofreram com a nova perspectiva de ensino e aprendizagem, sendo essas agora implementadas pelas tecnologias. Portanto, a utilização de metodologias ativas como recurso de ensino fora incorporada à prática docente e o retorno dos estudantes após acessar a sua comunidade também foram mediadas pelas tecnologias.

A prática docente universitária, por sua vez, exige do docente múltiplos saberes que perpassam o campo disciplinar articulados com os saberes pedagógicos e aqueles advindos da prática profissional (ALTHAUS; BAGGIO, 2017), exigindo do docente maleabilidade e capacidade de aprendizagem emergente na nova modalidade.

As metodologias ativas de ensino e aprendizagem por sua vez, são um recurso que vem se firmando como forma de aprofundar a aprendizagem, uma vez que tem como base o processo de mediação do conhecimento realizado pelo professor, problematizando, perguntando, intervindo, dialogando e ensinando, práticas que são dialógicas. O uso de metodologia ativa exige preparo prévio do estudante, estudo, desenvolvendo sua autonomia e o engajamento na atividade (ALTHAUS; BAGGIO, 2017). A aprendizagem é mais efetiva quando o estudante reflete e não apenas ouve uma explicação, especialmente quando essa atividade é feita em grupos, onde há possibilidade de compartilhamento dessas vivências.

Outro fator importante nesse processo é o uso das tecnologias, estas que por muitos anos foram vistas como um meio que tirava o ser humano do convívio social, durante a pandemia tornou-se um benefício coletivo que em virtude do isolamento social introduz às pessoas a relações de proximidades, permitindo que estas possam experimentar a unidade mesmo que de forma remota. Assim as tecnologias e os aparelhos tecnológicos se tornaram ferramentas pedagógicas (SANTOS JUNIOR, MONTEIRO, 2020; PEREIRA, MACHADO, TOLFO, 2020).

Almeida (2020) refere que as tecnologias foram os recursos que tornaram possível a continuidade dos estudos, mesmo em meio às dificuldades geradas por conexões de internet de baixa potência 
e o desconhecimento sobre o uso destes recursos, que viabilizassem a reprodução de metodologias já utilizadas em sala de aula. Salienta-se também que a IES em questão vem investindo em formação dos professores para atuar com esses recursos, processo que favorece a diversificação de metodologias recursos, tornando possível o aprendizado dos estudantes.

\section{CONCLUSÃo}

O primeiro semestre de 2020, devido à situação particular em razão da pandemia de COVID-19 não possibilitou a vivência in loco das atividades extensionistas. No entanto, a possibilidade de realização de materiais que podem ser disponibilizados nas redes de internet contribuiu para aumentar a abrangência da acessibilidade destas informações de promoção de saúde na comunidade. Também, a possibilidade de abrangência dos territórios de atuação (pela ampliação dos municípios e ESF envolvidos) permitiu a ampliação do espectro de visão da atuação profissional pelos estudantes, o que foi possível devido ao ensino remoto e disponibilidade das tecnologias.

Além disso, foi um desafio para os professores criar estratégias de ensino aprendizagem que motivassem os alunos a desenvolver as atividades propostas. Percebe-se, portanto, a capacidade de reinventar de cada docente e discente na busca por aprendizado, considerando que esse momento pandêmico trouxe para cada uma dessas disciplinas participantes do subprojeto de Ensino-Extensão Ações Extensionistas Interdisciplinares em Saúde a exigência de oferecer um ensino de qualidade com significado na troca de saberes tão caro para cada um daqueles que se envolvem no universo do ensino-aprendizado. Para isso, a experiência prática dos docentes que compõe as disciplinas foram elementos fundamentais na construção deste semestre.

\section{REFERÊNCIAS}

ALMEIDA, M.C.R. Práticas pedagógicas mediadas por tecnologias digitais em período de pandemia. Rev. Docência Ens. Sup., Belo Horizonte, v. 10, e024827, 2020.

ALTHAUS, M. T. M. BAGIO, V. A. As metodologias ativas e as aproximações entre o ensino e a aprendizagem na prática pedagógica universitária. Rev. Docência Ens. Sup., Belo Horizonte, v. 7, n. 2 , p. 79-96, jul./dez. 2017.

FARIAS, D. N.; RIBEIRO, K. S. Q. S.; ANJOS, U. U., BRITO, G. E. G. Interdisciplinaridade e interprofissionalidade na Estratégia Saúde da Família. Trab. educ. saúde, Rio de Janeiro, v. 16, n. 1, p. 141-162, abr. 2018. 
FEUERWERKER, Laura C. M.; CECÍLIO, Luiz C. O. O hospital e a formação em saúde: desafios atuais. Ciên \& Saú. Coletiva, Rio de Janeiro, v. 12, n. 4, p. 965-971, 2007.

LIMA, V. V. Espiral construtiva: uma metodologia ativa de ensino-aprendizagem. Rev. Interface, v. 21, n. 61, p. 421-434, 2017.

MATOS, E.; PIRES, D. E. P de; SOUSA, G. W. Relações de trabalho em equipes interdisciplinares: contribuições para novas formas de organização do trabalho em saúde. Rev. Bras. Enf. v. 63, n. 5, p. $775-781,2010$.

MORITA MC, KRIGER L. Mudanças nos cursos de Odontologia e a interação com o SUS. Revista ABENO, v. 4, n. 1, p.17-21, 2003.

PEREIRA, LA.; MACHADO, EM; TOLFO, K. Relação professor e estudante em tempos de pandemia. Rev. Franc. Edu. Santa Maria, v. 4, p. 14-24, 2020.

PRODANOV, C. C.; FREITAS, E. Metodologia do trabalho científico: métodos e técnicas da pesquisa e do trabalho. [recurso eletrônico] 2. ed. Novo Hamburgo: Feevale, 2013.

SANTOS JUNIOR, VERISSIMO BARROS DOS; MONTEIRO, JEAN CARLOS DA SILVA. Educação e Covid-19: As Tecnologias Digitais Mediando a Aprendizagem em Tempos de Pandemia. Rev. Encantar - Edu, Cult e Soc., Bom Jesus da Lapa, v. 2, p. 01-15, jan./dez. 2020. 